\title{
Assessment of Knowledge, Beliefs and Level of Internet Addiction among Nursing Students at Minia University
}

\author{
Shimaa Abd El-Razek Younis ${ }^{1}$, Eman Mohamed Mahfouz ${ }^{2}$, Yosria El-Sayed Hossien ${ }^{3}$
}

1. Assistant lecturer of Community Health Nursing, Faculty of Nursing, Minia University, Egypt.

2. Professor of Public Health medicine, Faculty of Medicine, Minia University, Egypt.

3. Professor of Community Health Nursing, Faculty of Nursing, Minia University, Egypt.

\begin{abstract}
Background: Worldwide, Internet addiction is a major and serious challenge. With uncontrolled use of the internet, university students may suffer from academic problems, distractions, and social isolation. Aim: This study aimed to assess knowledge, beliefs, and level of Internet addiction among nursing students at Minia University. The Health Belief Model was used as a theoretical framework in the study. Design: Descriptive research design. Setting: The study was conducted at the faculty of nursing at Minia University. Sample: Three hundred and seventy (370) students were included using a stratified random sample. Tools: Data collected using two tools, the 1 st tool was a selfadministered questionnaire based on The Health Belief Model to assess the students' knowledge and beliefs about Internet addiction, and the 2nd tool was the Arabic version of the Internet Addiction Test to assess the students' level of Internet addiction. Results: $91.1 \%$ of the participants had poor knowledge about Internet addiction. Regarding health beliefs toward IA, 66.5\% had low perceived susceptibility, $64.1 \%$ had low perceived severity, 53.5\% had high perceived barriers, $56.8 \%$ had high perceived benefits, $64.3 \%$ had low perceived cues to action, and $73 \%$ had low perceived self-efficacy. Concerning the level of Internet addiction, $44.6 \%$ had a mild level followed by $38.9 \%$ had a moderate level and 3.5\% had a severe level of Internet addiction. Conclusion: The majority of participants had poor knowledge and low Health Belief Model constructs toward Internet addiction except for perceived barriers. The majority had mild and moderate levels of Internet addiction and the minority had a severe Internet addiction. Recommendation: strategies should be developed to increase awareness and decrease the level of Internet addiction among university students.

Key words: Internet addiction, Beliefs, Knowledge, Level
\end{abstract}

\section{Introduction}

The Internet has become one of the most important tools for knowledge, work opportunities, education and amusement involving social media platforms and networking and is increasingly developed to be a structural element of our daily life (Thakur al., 2018). Over the past fifteen (15) years, Internet use has grown very fast: in current society about $40 \%$ of the global population is online ( Kuss et al., 2014). The growing popularity and frequency of internet use has resulted in the appearance of clinical conditions manifesting abuse symptoms identified as Internet addiction (IA) (Spada, 2014). Internet addiction is classically defined as a condition where an individual has impaired control of their internet use and proceed to use the internet too much to the point where he/she suffers problematic effects which ultimately have negative consequences on his/her life(Smyth et al., 2019). Internet addiction primarily put forward by Ivan Goldberg in 1995 and since then, it has become a social-psychological problem and a lot of researchers have been studying this topic (Dongyun et al., 2018; Wiederhold, 2018\& Griffiths, 2018). Even though IA was not formally added into the fifth edition of the Diagnostic and Statistical Manual of Mental Disorders (DSMV) in 2013, Internet Gaming Disorder (IGD) has been involved in sector III, highlighting the significance of this area for further study (Petry \& O'brien, 2013; Cho et al., 2014; Hahn et al., 2017\& Spada, 2014).

Assessment of knowledge and beliefs regarding safe usage of the internet is necessary. Ong and Tan (2014) in a study aimed to assess IA in young people showed that knowledge of IA among the public is a pertinent factor in the prevention efforts regarding IA. Maheri et al., (2017) showed that improving college students' knowledge and attitude about the addictive nature of the internet and side effects of IA are crucial for the prevention of IA(Maheri et $P$ a g e | 30 al., 2018). The role of the nurse is to contribute to the preventive and therapeutic intervention to face this phenomenon. The nurse should help students to understand the effects of excessive internet usage on themselves physically and mentally and how to overcome these impacts of internet addiction(Hamzaa, 2017). Considering the globalization and the complexity of IA community health nurses must establish an effective program for the management of the addiction as well as the daily problems that such condition raises. Within the clinical context of mental health, nurses can have an effective role not only in the assessment, diagnosis, and treatment of IA but in the prevention of that phenomenon as well (Fradelos et al., 2016).

\section{Significance of The study}

Internet addiction (IA) is a worldwide phenomenon with different levels and it ranges from five to twenty-five percent among students in the united states (US), China, South Korea, England, Australia, Taiwan, Japan, and other countries in Eastern and Western Europe (Maheri et al., 2017). Internet World Stats revealed that Egypt has the secondhighest number of internet users in Africa after Nigeria. On average, Egyptians stay 26 hours a week on the internet according to the MidEast Media Survey 'Media Use in the Middle East' in 2017 (Eltigani, 2019). University students are particularly at risk for encountering dependence on the Internet, greater than other segments of the community. This can be attributed to numerous factors as the availability of time, easiness of use, limitless access to the Internet and limited or no familial supervision (MMIN, 2017). Assessment of IA among university students is a pertinent factor for its prevention effort. 
Aim of the Study

The current study aimed to assess knowledge, beliefs and level of internet addiction among nursing students at Minia University

\section{Theoretical Framework}

One of the most commonly applied models in explaining and adopting healthy behaviors such as the protective behavior of addiction is the Health Belief Model (HBM) (Zadeh et al., 2014). The HBM was created in the US in 1950 by the Department of Public Health Service to understand the reasons for the ineffectiveness of public health services directed toward the prevention of health problems. The application of the model after that extended for comprehending the adherence with clinical remedy (Orji et al., 2012). Health Belief Model explains that health-related behaviors of people are based on their perceived susceptibility, perceived severity, perceived benefits, perceived barriers, cues to action, and perceived self-efficacy (Zadeh et al., 2014).

\section{Subjects and Methods}

Research Design

Descriptive research design was utilized in the current study.

\section{Setting:}

The study was conducted at faculty of nursing at Minia University.

\section{Sample size}

Sample size was calculated based on Cochran formula, (1963) while $\mathbf{n}=\mathbf{t}^{2} \times \mathbf{p}(\mathbf{1}-\mathbf{p}) / \mathbf{m}^{2}$ considering $41.5 \%$ prevalence rate of internet addiction according to Abdelghani, $M$ et al., (2018).

\section{Inclusion criteria for the study sample}

(1) Undergraduate students at faculty of nursing at Minia University

(2) Currently using the internet for at least 3 hours/day and in the past 6 months

\section{Study Tools}

Tool 1: A self-administered questionnaire developed by the researcher based on $\mathrm{HBM}$ and consisted of 4 parts as following:

Part I: sociodemographic data of the students such as: Age, sex, faculty grade, residence, parents' education, family income, quality of relation with parents and friends, residence during studying, smoking status and academic average.

Part II: the student's knowledge about IA such as: definition, signs, causes, types of IA, its physical, psychological and social effects, and management of IA.

\section{Scoring system}

The scores for knowledge part of the questionnaire were calculated based on one point (1) for the correct answer and zero (0) for the wrong answer or don't know respectively. Therefore the participants were considered to have a very good level of knowledge if the total score $>75 \%$, good if the total score ranged from $60-75 \%$ and poor if the total score $<$ 60\% MMIN, M. (2017).
Part III: A five-point Likert scale based on HBM assessed the students' beliefs toward IA. The scale consisted of six subscales as following: Perceived susceptibility (one question) and perceived severity (5 questions), perceived barriers ( 9 questions), perceived benefits (6 question), cues to action ( 2 questions) and perceived self-efficacy (5questions).

\section{Scoring system}

The statements of this part were scored on a fivepoint likert-type scale as following: strongly disagree (1), disagree (2), neutral (3), agree (4), and strongly agree (5). The entire score for each subscale was calculated by summing up of all of its statements. With summed scores $>60 \%$ indicates a higher level of the HBM subscale/construct while a summed score $\leq 60 \%$ indicates a lower level of the subscale for each one.

Tool 2: The Arabic version of Internet Addiction test (IAT): It is a self-rated scale developed by Young (1998) to assess the existence and severity of IA. This tool consists of twenty (20) items; These 20 items involve distraction, compulsivity, and dependency. The items also assess conflicts in personal, social, or occupational life that may arise from the addictive use of the internet. The Arabic version of IAT has been validated in a study conducted in Lebanon by Hawi (2013).

\section{Scoring system}

The statements of the IA test were scored on a fivepoint Likert-type scale, rarely (1), occasionally (2), frequently (3), often (4), always (5). Sum of the scores that ranged from 0 to 30 points indicated a normal level of internet usage; scores of 31 to 49 reflected a mild level of IA; scores of 50 to 79 indicated a moderate level, and scores of 80 to 100 indicated severe internet dependence (Young, 1998).

\section{Content Validity of the Tools}

The content validity of the study tools was tested by five experts in community health nursing. The tools were examined for content coverage, sequence of items, clarity, relevance, applicability, words length, format, and overall appearance. Based on experts ' comments, recommendations and modifications were made.

\section{Reliability of the Tools}

Reliability of the study questionnaire was calculated using Cronbach's alpha. Based on data analysis, coefficient alpha for the knowledge part was 0.78 , perceived severity was 0.81 , perceived barriers was 0.82 , perceived benefits was 0.76 , cues to action was 0.68 , self efficacy was 0.92 , for all the subscales of the HBM was 0.080, and for the entire questionnaire was 0.70 . As regard to the reliability of the IAT, it was 0.93 .

\section{Procedure}

Before conducting the study an official permission was taken from the dean of the faculty of nursing to conduct the study. The interview with the students of each grade was held at their specialized faculty class. The researcher first introduced herself to the students, explained to them the purposes of the study briefly and an oral consent for participation was obtained. The tools of the study were filled 
by the students and aided by the researcher. The time required to fill the questionnaire was about 15 minutes.

\section{Pilot study}

It was applied on $10 \%$ of the calculated sample to assess the validity of the questionnaire and to assess acceptability of the students to the topic of the research. The results of pilot study were included in the final results of the research as there were no major modifications were done in the tools of the study

\section{Statistical Analysis}

Data entry and statistical analysis were done using SPSS 24.0 statistical software package. Data presented using descriptive statistics in the form of frequencies and percentages for qualitative variables, and means and standard deviations for quantitative variables. The Chi square used in tests of relationship. Probability (P-value) less than 0.05 was considered significant. $(\mathrm{p}<0.05)$.

\section{Ethical considerations}

A written approval obtained from the ethics and research committee of the faculty of nursing at Minia University. Oral consent obtained from students after explaining the nature and objectives of the study to gain their cooperation. Each assessment sheet was coded for the purpose of privacy and confidentiality. Participants were free to withdraw from the study at any time.

\section{Results}

Table (1) Distribution of the studied Minia university nursing students according to their socioemographic characteristics in the academic year of $2018 / 2019(n=370)$

\begin{tabular}{|c|c|c|}
\hline Socio-demographic characteristics & No & Percent $\%$ \\
\hline Age & & \\
\hline $18-21$ & 244 & 65.9 \\
\hline $22-24$ & 126 & 34.1 \\
\hline \multicolumn{3}{|l|}{ Mean \pm SD $20.78 \pm 1.30$} \\
\hline \multicolumn{3}{|l|}{ Gender } \\
\hline Male & 150 & 40.5 \\
\hline Female & 220 & 59.5 \\
\hline \multicolumn{3}{|l|}{ Faculty grade } \\
\hline 1st year & 89 & 24.0 \\
\hline 2nd year & 85 & 23.0 \\
\hline 3rd year & 111 & 30.0 \\
\hline 4th year & 85 & 23.0 \\
\hline \multicolumn{3}{|l|}{ Residence } \\
\hline Rural & 278 & 75.1 \\
\hline Urban & 92 & 24.9 \\
\hline \multicolumn{3}{|l|}{ Quality of relation with parents } \\
\hline Good & 358 & 96.8 \\
\hline Poor & 12 & 3.2 \\
\hline \multicolumn{3}{|l|}{ Residence during study } \\
\hline with family & 231 & 62.4 \\
\hline away from family & 139 & 37.6 \\
\hline \multicolumn{3}{|l|}{ Smoking } \\
\hline Smoker & 20 & 5.4 \\
\hline Non smoker & 350 & 94.6 \\
\hline \multicolumn{3}{|l|}{ Academic performance } \\
\hline Excellent & 75 & 20.2 \\
\hline Very good & 156 & 42.2 \\
\hline Good & 105 & 28.4 \\
\hline Pass or weak & 34 & 9.2 \\
\hline \multicolumn{3}{|l|}{ Father education } \\
\hline Does not read or write & 52 & 14.1 \\
\hline Primary & 47 & 12.7 \\
\hline Preparatory & 35 & 9.5 \\
\hline Secondary & 128 & 34.6 \\
\hline University & 83 & 22.4 \\
\hline Post university studies & 25 & 6.8 \\
\hline \multicolumn{3}{|l|}{ Mother education } \\
\hline Does not read or write & 116 & 31.4 \\
\hline Primary & 44 & 11.9 \\
\hline Preparatory & 41 & 11.1 \\
\hline Secondary & 109 & 29.5 \\
\hline University & 48 & 13.0 \\
\hline Post university studies & 12 & 3.2 \\
\hline \multicolumn{3}{|l|}{ Family income/month } \\
\hline less than 2000 L.E & 166 & 44.9 \\
\hline 2000-3000 L.E & 158 & 42.7 \\
\hline More than 3000 L.E & 46 & 12.4 \\
\hline
\end{tabular}

Table (1) shows that $65.9 \%$ of the participants are in the age group $18-21$ yrs with a mean score \pm SD $20.78 \pm 1.30,59.5 \%$ of the participants are females, $75.1 \%$ live in rural areas, $96.8 \%$ have a good relationship with their parents, $62.4 \%$ are residents with their parents during the study, 5.4\% are smokers, and $42.2 \%$ their academic performance is very good. The table also shows that $34.6 \%$ of the participants their fathers' education is a secondary education, $31.4 \%$ their mothers don't read or write, $44.9 \%$ of the participants their monthly family income is less than 2000 L.E 
Minia Scientific Nursing Journal (Print - ISSN 2537-012X) (Online - ISSN 2785-9797) Vol. (8) No. (1) December 2020

Table (2) Distribution of the studied Minia University nursing students according to their knowledge about definition and signs of IA $(\mathbf{N}=\mathbf{3 7 0})$

\begin{tabular}{|c|c|c|}
\hline Item & No & $\%$ \\
\hline $\begin{array}{ll}\text { Definition } \\
\qquad & \text { Complete answer } \\
\bullet & \text { Incomplete answer } \\
\text { - } & \text { I don't know }\end{array}$ & $\begin{array}{c}96 \\
258 \\
16\end{array}$ & $\begin{array}{c}25.9 \\
69.7 \\
4.3\end{array}$ \\
\hline $\begin{array}{cl}\text { Signs of IA } & \\
\text { - } & \text { Irritability during withdrawal } \\
\text { - } & \text { Jeopardizing a significant relationship, or responsibilities } \\
\text { - } & \text { Loss of sense of time during use } \\
\text { - } & \text { Check electronic notifications } \\
\text { - } & \text { Preoccupation with the internet } \\
\text { - } & \text { Failure to reduce time of use } \\
\text { - } & \text { I don't know }\end{array}$ & $\begin{array}{c}163 \\
214 \\
\\
206 \\
114 \\
105 \\
185 \\
27\end{array}$ & $\begin{array}{c}44.1 \\
57.8 \\
55.7 \\
30.8 \\
28.4 \\
50.0 \\
7.3\end{array}$ \\
\hline
\end{tabular}

\section{"Mutual exclusive more than one answer}

Table (2) shows that $69.7 \%$ of the participants' definition of IA is incomplete while $4.3 \%$ don't know the definition of IA. Regarding knowledge about signs of IA $57.8 \%$ mention jeopardizing a significant relationship or responsibilities as a sign of IA while $7.3 \%$ doesn't know any signs of IA.

Table (3) Distribution of the studied Minia University nursing according to their knowledge about causes and social effects of IA (n=370)

\begin{tabular}{|c|c|c|}
\hline Item & $\begin{array}{l}\text { No } \\
\end{array}$ & $\%$ \\
\hline $\begin{array}{cl}\text { Causes }^{\#} & \\
\text { - } & \text { Personal privacy } \\
\text { - } & \text { Emotional relief } \\
\text { - } & \text { Fscape from reality } \\
\text { - } & \text { Feeling lonely boredom } \\
\text { - } & \text { Easy access } \\
\text { - } & \text { I don't know }\end{array}$ & $\begin{array}{c}78 \\
164 \\
189 \\
202 \\
141 \\
90 \\
21\end{array}$ & $\begin{array}{c}21.2 \\
44.6 \\
51.4 \\
54.9 \\
38.3 \\
24.5 \\
5.7\end{array}$ \\
\hline $\begin{array}{cl}\text { Social Effects } & \\
\text { - } & \text { Low academic performance } \\
\text { - } & \text { Family disconnection } \\
\text { - } & \text { low productivity of work } \\
\text { - I don't know }\end{array}$ & $\begin{array}{c}235 \\
87 \\
169 \\
68\end{array}$ & $\begin{array}{l}63.7 \\
23.6 \\
45.8 \\
18.4\end{array}$ \\
\hline
\end{tabular}

\#Mutual exclusive more than one answer

Table (3) shows that $54.9 \%$ of the participants mention free time and boredom as a cause of IA while 5.7\% don't know causes of IA. In relation to knowledge about social effects of IA, $63.7 \%$ mention low academic performance while $18.4 \%$ don't know its social effect

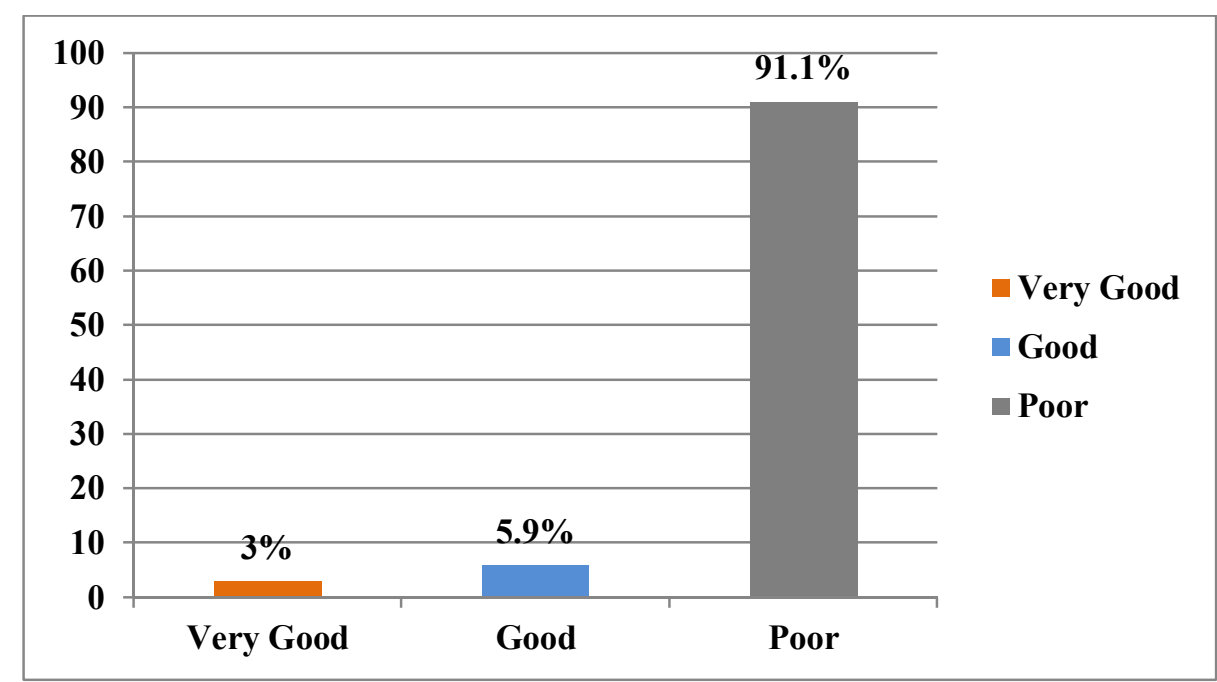

Fig. (1) Distribution of the studied Minia university nursing students according to their total level of knowledge about IA

Table (4a) Distribution of the studied Minia University nursing students according to their health beliefs toward IA (n=370)

\begin{tabular}{|c|c|c|c|c|c|c|c|c|c|c|}
\hline \multirow[t]{2}{*}{ Item } & \multicolumn{2}{|c|}{$\begin{array}{l}\text { Strongly } \\
\text { Agree }\end{array}$} & \multicolumn{2}{|c|}{ Agree } & \multicolumn{2}{|c|}{ Neutral } & \multicolumn{2}{|c|}{ Disagree } & \multicolumn{2}{|c|}{$\begin{array}{l}\text { Strongly } \\
\text { disagree }\end{array}$} \\
\hline & No & $\%$ & No & $\%$ & No & $\%$ & No & $\%$ & No & $\%$ \\
\hline $\begin{array}{l}\text { Perceived susceptibility } \\
\text { Likelihood o be internet addict }\end{array}$ & 42 & 11.4 & 82 & 22.2 & 103 & 27.8 & 107 & 28.9 & 36 & 9.7 \\
\hline $\begin{array}{l}\text { Perceived severity } \\
\text { IA is a serious disorder }\end{array}$ & 35 & 9.5 & 60 & 16.2 & 74 & 20 & 160 & 43.2 & 41 & 11.1 \\
\hline Thinking of IA is a restless issue? & 16 & 4.3 & 70 & 18.9 & 76 & 20.5 & 168 & 45.4 & 40 & 10.8 \\
\hline
\end{tabular}


Minia Scientific Nursing Journal (Print - ISSN 2537-012X) (Online - ISSN 2785-9797) Vol. (8) No. (1) December 2020

\begin{tabular}{|c|c|c|c|c|c|c|c|c|c|c|}
\hline \multirow[t]{2}{*}{ Item } & \multicolumn{2}{|c|}{$\begin{array}{c}\text { Strongly } \\
\text { Agree }\end{array}$} & \multicolumn{2}{|c|}{ Agree } & \multicolumn{2}{|c|}{ Neutral } & \multicolumn{2}{|c|}{ Disagree } & \multicolumn{2}{|c|}{$\begin{array}{l}\text { Strongly } \\
\text { disagree }\end{array}$} \\
\hline & No & $\%$ & No & $\%$ & No & $\%$ & No & $\%$ & No & $\%$ \\
\hline IA negatively affect my health & 27 & 7.3 & 70 & 18.9 & 74 & 20 & 161 & 43.5 & 38 & 10.3 \\
\hline IA negatively affect me academically & 68 & 18.4 & 154 & 41.6 & 72 & 19.5 & 55 & 14.9 & 21 & 5.7 \\
\hline IA may socially isolate me & 55 & 14.9 & 102 & 27.6 & 92 & 24.9 & 82 & 22.2 & 39 & 10.5 \\
\hline $\begin{array}{l}\text { Perceived barriers } \\
\text { limited social connection }\end{array}$ & 78 & 21.1 & 98 & 26.5 & 76 & 20.5 & 92 & 24.9 & 26 & 7.0 \\
\hline Life seems boring without internet & 54 & 14.6 & 116 & 31.4 & 68 & 18.4 & 84 & 22.7 & 48 & 13.0 \\
\hline Feeling lost without internet & 60 & 16.2 & 68 & 18.4 & 80 & 21.6 & 107 & 28.9 & 55 & 14.9 \\
\hline Being an old fashioned person & 61 & 16.5 & 95 & 25.7 & 55 & 14.9 & 103 & 27.8 & 56 & 15.1 \\
\hline No encouragement to $\downarrow$ online time & 58 & 15.7 & 92 & 24.9 & 61 & 16.5 & 126 & 34.1 & 33 & 8.9 \\
\hline Feeling lonely without internet & 76 & 20.5 & 88 & 23.8 & 82 & 22.2 & 87 & 23.5 & 37 & 10 \\
\hline Negative effect on self-esteem & 47 & 12.7 & 37 & 10 & 69 & 18.6 & 119 & 32.2 & 98 & 26.5 \\
\hline No other way to relieve stress & 102 & 27.6 & 79 & 21.4 & 50 & 13.5 & 86 & 23.2 & 53 & 14.3 \\
\hline No help in decision making without internet & 66 & 17.8 & 82 & 22.2 & 70 & 18.9 & 104 & 28.1 & 48 & 13 \\
\hline
\end{tabular}

Table (4a) shows that $28.9 \%$ of the participants disagree they are susceptible to IA. As regards to their Perceived severity of IA, $43.2 \%$ disagree that IA is a serious disease of the era. In the same domain of perceived severity, $45.4 \%$ disagree that thinking about the negative effects of IA on health is a restless issue, $43.5 \%$ disagree that IA may negatively affect their health, 41.6 agree that IA may negatively affect their academic performance, and $27.6 \%$ of the participants agree that IA may socially isolate them from their family.

Regarding the participants' perceived barriers toward reducing internet use, the same table shows that $26.5 \%$ agree that reducing internet time limit their social contact with friends and relatives, 31.4 agree that life seems boring without internet, $28.9 \%$ disagree they will feel lost if they reduce their internet time, $27.8 \%$ disagree that they will be an old fashioned if they reduced their internet time, $34.1 \%$ disagree that nobody encourages them to reduce their internet time, $23.8 \%$ agree they will feel lonely if reduce their internet time, $32.2 \%$ disagree that reducing internet time may negatively affect their self-esteem, $27.6 \%$ strongly agree that there is no other way to relieve stress if they reduced their internet use, and $28.1 \%$ agree that nobody will help them in their decision making if they reduce internet use.

Table (4b) Distribution of the studied Minia University nursing students according their health beliefs toward IA (n=370)

\begin{tabular}{|c|c|c|c|c|c|c|c|c|c|c|}
\hline \multirow{2}{*}{ Item } & \multicolumn{2}{|c|}{ Strongly Agree } & \multicolumn{2}{|c|}{ Agree } & \multicolumn{2}{|c|}{ Neutral } & \multicolumn{2}{|c|}{ Disagree } & \multicolumn{2}{|c|}{ Strongly disagree } \\
\hline & No & $\%$ & No & $\%$ & No & $\%$ & No & $\%$ & No & $\%$ \\
\hline $\begin{array}{l}\text { Perceived benefits } \\
1 \text {-Focusing on important issues }\end{array}$ & 49 & 13.2 & 104 & 28.1 & 112 & 30.3 & 54 & 14.6 & 51 & 13.8 \\
\hline 3- Good relation with family/friends & 45 & 12.2 & 93 & 25.1 & 108 & 29.2 & 96 & 25.9 & 28 & 7.6 \\
\hline 4- Self satisfied with reduced use & 36 & 9.7 & 141 & 38.1 & 122 & 33 & 48 & 13 & 23 & 6.2 \\
\hline 5- Enjoying personal privacy & 49 & 13.2 & 103 & 27.8 & 121 & 32.7 & 72 & 19.5 & 25 & 6.8 \\
\hline 6- Positive effects on health & 27 & 7.3 & 68 & 18.4 & 75 & 20.3 & 162 & 43.8 & 38 & 10.3 \\
\hline $\begin{array}{l}\text { Cues to action } \\
1-\text { Cues to action from parents }\end{array}$ & 43 & 11.6 & 142 & 38.4 & 27 & 7.3 & 97 & 26.2 & 61 & 16.5 \\
\hline 2- Cues to action from teachers & 26 & 7.0 & 93 & 25.1 & 37 & 10 & 117 & 31.6 & 97 & 26.2 \\
\hline $\begin{array}{l}\text { Perceived self-Efficacy } \\
\text { 1- Ability to reduce internet time? }\end{array}$ & 25 & 6.8 & 78 & 21.1 & 91 & 24.6 & 85 & 23.0 & 91 & 24.6 \\
\hline 2- Easiness to reduce internet time & 23 & 6.2 & 58 & 15.7 & 40 & 10.8 & 98 & 26.5 & 151 & 40.8 \\
\hline 3- Having a plenty of ideas to reduce internet time & 19 & 5.1 & 51 & 13.8 & 83 & 22.4 & 103 & 27.8 & 114 & 30.8 \\
\hline $\begin{array}{l}\text { 4- If I worked hard on reducing time of internet } \\
\text { use, I would do it. }\end{array}$ & 24 & 6.5 & 60 & 16.2 & 82 & 22.2 & 99 & 26.8 & 105 & 28.4 \\
\hline $\begin{array}{l}\text { 5-I intention to reduce daily hours of } \\
\text { internet time }\end{array}$ & 33 & 8.9 & 90 & 24.3 & 114 & 30.8 & 62 & 16.8 & 71 & 19.2 \\
\hline
\end{tabular}

Table (4b) shows that $30.2 \%$ of the participants are neutral about giving priority to important life issues as perceived benefits of reducing internet, $42.2 \%$ agree that reducing the time of using the internet has a positive effect on their academic achievements, $29.2 \%$ are neutral about the benefit of improving their family and friends relation quality if they reduce internet time, $38.1 \%$ agree that reducing their internet time will make them self satisfied, $32.7 \%$ are neutral about enjoying more personal privacy as a benefit of reducing internet time, and $43.8 \%$ disagree that reducing internet time has positive effects on their health.

As regards to cues to action toward IA, the same table shows that $38.4 \%$ of the participants agree that their parents asking for reducing internet time, $31.6 \%$ disagree that their teachers ask them to reduce their internet time. Concerning perceived self-efficacy toward reducing internet time, $24.6 \%$ are neutral that they have the ability finding suitable ways to reduce internet usage, $40.8 \%$ strongly disagree that it is easy to reduce internet time, $30.8 \%$ disagree that they have a plenty of ideas how to reduce internet time, and $30.8 \%$ are neutral about their intention to reduce daily hours of internet use. 


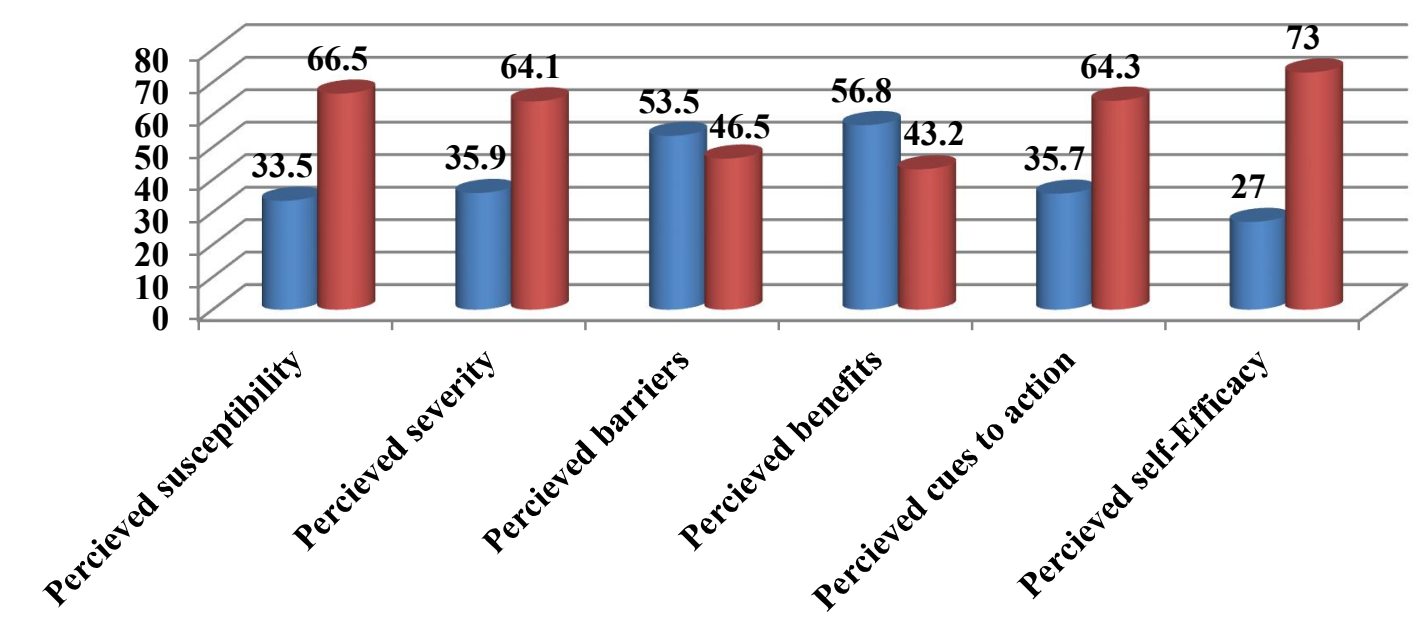

High

- Low

Fig. (2) Distribution of the studied Minia University nursing students' level of health beliefs toward IA

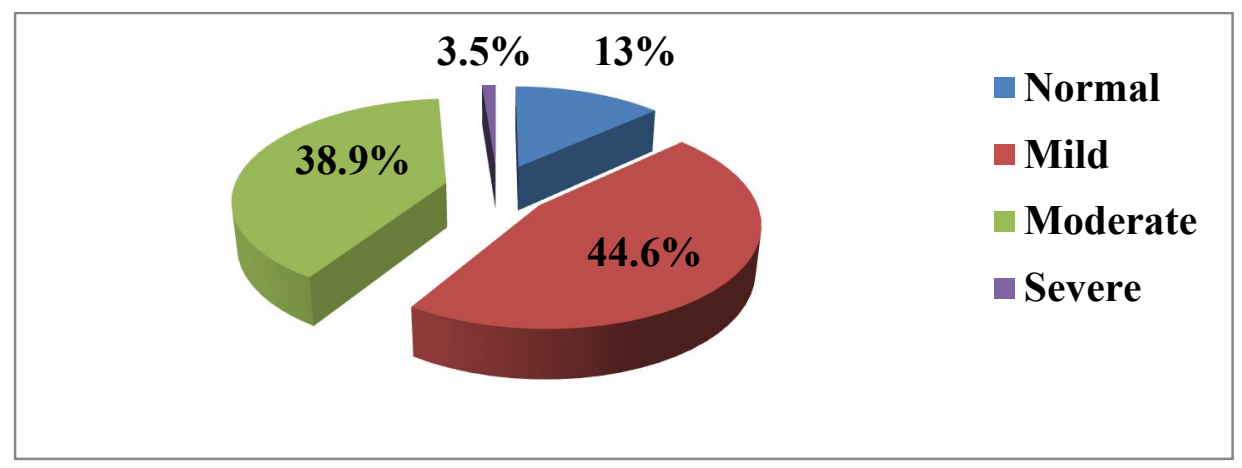

Fig. (3) Level of IA among the studied Minia university nursing students, according to Young's IAT (1998)

Fig. (3) Illustrates that, $44.6 \%$ of the participants had a mild level of IA followed by $38.9 \%$ had a moderate level, and $3.5 \%$ had a severe level of IA.

Table (5): Relation between the studied Minia University nursing students' total level of IA and their socio-demographic data $(n=370)$

\begin{tabular}{|c|c|c|c|c|c|c|c|c|c|c|}
\hline \multirow{3}{*}{ Variables } & \multicolumn{8}{|c|}{ Level of IA $(n=370)$} & \multirow{3}{*}{$\mathbf{X}^{2}$} & \multirow{3}{*}{$\mathbf{P}$} \\
\hline & \multicolumn{2}{|c|}{$\begin{array}{l}\text { Normal } \\
(\mathrm{n}=48)\end{array}$} & \multicolumn{2}{|c|}{$\begin{array}{c}\text { Mild } \\
(\mathrm{n}=165)\end{array}$} & \multicolumn{2}{|c|}{$\begin{array}{c}\text { Moderate } \\
(\mathrm{n}=144)\end{array}$} & \multicolumn{2}{|c|}{$\begin{array}{l}\text { Severe } \\
(\mathrm{n}=13)\end{array}$} & & \\
\hline & No & $\%$ & No & $\%$ & No & $\%$ & No & $\%$ & & \\
\hline $\begin{array}{l}\text { Age(year) } \\
-18-21 \\
=22-24\end{array}$ & $\begin{array}{l}35 \\
13\end{array}$ & $\begin{array}{l}72.9 \\
27.1\end{array}$ & $\begin{array}{c}104 \\
61\end{array}$ & $\begin{array}{l}63.0 \\
37.0\end{array}$ & $\begin{array}{l}98 \\
46\end{array}$ & $\begin{array}{l}68.1 \\
31.9\end{array}$ & $\begin{array}{l}7 \\
6\end{array}$ & $\begin{array}{l}53.8 \\
46.2\end{array}$ & 2.7 & 0.4 \\
\hline $\begin{array}{l}\text { Gender } \\
\text {-Male } \\
\text {-Female }\end{array}$ & $\begin{array}{c}7 \\
41\end{array}$ & $\begin{array}{l}14.6 \\
85.4\end{array}$ & $\begin{array}{c}64 \\
101\end{array}$ & $\begin{array}{l}38.8 \\
61.2\end{array}$ & $\begin{array}{l}69 \\
75\end{array}$ & $\begin{array}{l}47.9 \\
52.1\end{array}$ & $\begin{array}{c}10 \\
3\end{array}$ & $\begin{array}{l}76.9 \\
23.1\end{array}$ & 24.1 & $0.001 *$ \\
\hline $\begin{array}{l}\text { Faculty Grade } \\
-1^{\text {st }} \text { year } \\
-2^{\text {nd }} \text { year } \\
-3^{\text {rd }} \text { year } \\
-4^{\text {th }} \text { year } \\
\end{array}$ & $\begin{array}{c}9 \\
13 \\
17 \\
9 \\
\end{array}$ & $\begin{array}{l}18.8 \\
27.1 \\
35.4 \\
18.8 \\
\end{array}$ & $\begin{array}{l}28 \\
49 \\
41 \\
47 \\
\end{array}$ & $\begin{array}{l}17.0 \\
29.7 \\
24.8 \\
28.5 \\
\end{array}$ & $\begin{array}{l}47 \\
22 \\
50 \\
25\end{array}$ & $\begin{array}{l}32.6 \\
15.3 \\
34.7 \\
17.4 \\
\end{array}$ & $\begin{array}{l}5 \\
1 \\
3 \\
4 \\
\end{array}$ & $\begin{array}{c}38.5 \\
7.7 \\
23.1 \\
30.8 \\
\end{array}$ & 26.2 & $0.002 *$ \\
\hline $\begin{array}{l}\text { Residence } \\
\text {-Rural } \\
\text {-Urban } \\
\end{array}$ & $\begin{array}{l}34 \\
14 \\
\end{array}$ & $\begin{array}{l}70.8 \\
29.2 \\
\end{array}$ & $\begin{array}{c}128 \\
37 \\
\end{array}$ & $\begin{array}{l}77.6 \\
22.4 \\
\end{array}$ & $\begin{array}{c}110 \\
34 \\
\end{array}$ & $\begin{array}{l}76.4 \\
23.6 \\
\end{array}$ & $\begin{array}{l}6 \\
7 \\
\end{array}$ & $\begin{array}{l}46.2 \\
53.8 \\
\end{array}$ & 6.9 & 0.07 \\
\hline $\begin{array}{l}\text { Father Education } \\
\text {-Do not read or write } \\
\text {-Primary } \\
\text {-Preparatory } \\
\text {-Secondary } \\
\text {-University } \\
\text {-Post university }\end{array}$ & $\begin{array}{c}9 \\
5 \\
7 \\
16 \\
8 \\
3\end{array}$ & $\begin{array}{c}18.8 \\
10.4 \\
14.6 \\
33.3 \\
16.7 \\
6.3\end{array}$ & $\begin{array}{l}18 \\
24 \\
11 \\
67 \\
31 \\
14\end{array}$ & $\begin{array}{c}10.9 \\
14.5 \\
6.7 \\
40.6 \\
18.8 \\
8.5\end{array}$ & $\begin{array}{c}24 \\
16 \\
16 \\
41 \\
41 \\
6\end{array}$ & $\begin{array}{c}16.7 \\
11.1 \\
11.1 \\
28.5 \\
28.5 \\
4.2\end{array}$ & $\begin{array}{l}1 \\
2 \\
1 \\
4 \\
3 \\
2\end{array}$ & $\begin{array}{c}7.7 \\
15.4 \\
7.7 \\
30.8 \\
23.1 \\
15.4\end{array}$ & 18.1 & 0.2 \\
\hline $\begin{array}{l}\text { Mother Education } \\
\text {-Do not read or write } \\
\text {-Primary } \\
\text {-Preparatory }\end{array}$ & $\begin{array}{c}14 \\
10 \\
5 \\
12 \\
\end{array}$ & $\begin{array}{l}29.2 \\
20.8 \\
10.4 \\
25.0 \\
\end{array}$ & $\begin{array}{l}48 \\
22 \\
20 \\
55 \\
\end{array}$ & $\begin{array}{l}29.1 \\
13.3 \\
12.1 \\
33.3 \\
\end{array}$ & $\begin{array}{l}49 \\
12 \\
15 \\
39 \\
\end{array}$ & $\begin{array}{c}34.0 \\
8.3 \\
10.4 \\
27.1 \\
\end{array}$ & $\begin{array}{l}5 \\
0 \\
1 \\
3 \\
\end{array}$ & $\begin{array}{c}38.5 \\
0.0 \\
7.7 \\
23.1 \\
\end{array}$ & 14.9 & 0.4 \\
\hline
\end{tabular}




\begin{tabular}{|c|c|c|c|c|c|c|c|c|c|c|}
\hline \multirow{3}{*}{ Variables } & \multicolumn{8}{|c|}{ Level of IA $(n=370)$} & \multirow{3}{*}{$\mathbf{X}^{2}$} & \multirow{3}{*}{$\mathbf{P}$} \\
\hline & \multicolumn{2}{|c|}{$\begin{array}{l}\text { Normal } \\
(n=48)\end{array}$} & \multicolumn{2}{|c|}{$\begin{array}{c}\text { Mild } \\
(n=165)\end{array}$} & \multicolumn{2}{|c|}{$\begin{array}{c}\text { Moderate } \\
(n=144)\end{array}$} & \multicolumn{2}{|c|}{$\begin{array}{l}\text { Severe } \\
(n=13)\end{array}$} & & \\
\hline & No & $\%$ & No & $\%$ & No & $\%$ & No & $\%$ & & \\
\hline -Secondary & 6 & 12.5 & 15 & 9.1 & 24 & 16.7 & 3 & 23.1 & & \\
\hline $\begin{array}{l}\text {-University } \\
\text {-Post university }\end{array}$ & 1 & 2.1 & 5 & 3.0 & 5 & 3.5 & 1 & 7.7 & & \\
\hline Family income/month & & & & & & & & & & \\
\hline - less than 2000 L.E & 21 & 43.8 & 75 & 45.5 & 69 & 47.9 & 1 & 7.7 & & \\
\hline -2000-3000 L.E & 25 & 52.1 & 71 & 43.0 & 51 & 35.4 & 11 & 84.6 & 17.5 & $0.008^{*}$ \\
\hline -more than 3000 L.E & 2 & 4.2 & 19 & 11.5 & 24 & 16.7 & 1 & & & \\
\hline Residence during study & & & & & & & & & & \\
\hline -with family & 29 & 60.4 & 109 & 66.1 & 85 & 59.0 & 8 & 61.5 & 1.7 & 0.6 \\
\hline -away from family & 19 & 39.6 & 56 & 33.9 & 59 & 59.0 & 5 & 38.5 & & \\
\hline Smoking & & & & & & & & & & \\
\hline -Smoker & 0 & 0.0 & 8 & 4.8 & 9 & 6.3 & 3 & 23.1 & 10.9 & $0.01 *$ \\
\hline -Non smoker & 48 & 100.0 & 157 & 95.2 & 135 & 93.8 & 10 & 76.9 & & \\
\hline Academic performance & & & & & & & & & & \\
\hline -Excellent & 16 & 33.3 & 32 & 19.4 & 27 & 18.8 & 0 & 0.0 & & \\
\hline -Very good & 20 & 41.7 & 81 & 49.1 & 52 & 36.1 & 3 & 23.1 & 39.6 & $0.001 *$ \\
\hline -Good & 9 & 18.8 & 45 & 27.3 & 47 & 32.6 & 4 & 30.8 & & \\
\hline -Pass or weak & 3 & 6.3 & 7 & 4.2 & 18 & 12.5 & 0 & 40.2 & & \\
\hline
\end{tabular}

\section{* Statistical significant difference, Chi-squared test.}

Table (6) shows that there are significant statistical differences between the level of IA and gender of the participants while males have a significant sever level of IA compared to females where the p-value is 0.001 . The same table shows that there are significant statistical differences between the level of IA and faculty grade of the participants where the p-value is 0.002 . An additional statistically significant difference is found between the level of IA and monthly family income of the participants while participants with a monthly family income ranges from 2000-3000 LE have a significant severe level of IA compared to others where the p-value is 0.008 . Another statistically significant dereference is found between the level of IA and smoking status of the participants while non-smokers have a significant severe level of IA compared to smokers where the p-value is 0.01 . The same table shows that there are significant statistical differences between the level of IA and academic level of the participants while participants whose academic performance is pass/weak have a significant severe level of IA compared to those with higher academic performance where the $\mathrm{p}$-value is 0.001 .

\section{Discussion}

One of the significant attributes of the current societies is the increased media utilization particularly the internet. Important benefits of the internet shall not delude us from the rising inclination of IA (Maheri et al., 2018). The current study aimed to assess knowledge, beliefs, and level of IA among nursing students at Minia University.

As regards to the level of knowledge about IA among the participants, the current study revealed that the majority (91.1\%) of the participants had a poor level of knowledge about IA followed by $5.9 \%$ had good knowledge, and the minority (3\%) had very good knowledge. This result agreed with Chander (2019) who revealed that the majority (78.33\%) of the participants had a poor level of knowledge and less than one quarter $(21.67 \%)$ had a good level of knowledge about the negative effects of IA. Similar to the current study MMIN (2017) found that the majority (58\%) had an average knowledge, followed by about one-third (34\%) had good knowledge regarding using of the internet. A Previous study by Zadeh et al. (2014) reported that knowledge is essential for admitting healthy behavior such as addiction protective behaviors. Also knowledge about the negative effects of addictive behaviors can save students against it. Thus, raising knowledge of university students about the addictive nature of the internet and side effects of IA is necessary for changing their IA behavior.

As regard to health beliefs toward IA among the participants, the current study showed that less than half (38.6\%) of the participants disagreed with their susceptibility to IA. similarly, Wang et al. (2016) revealed that more than half $(53.2 \%)$ of the participants disagreed with their susceptibility to IA. Concerning the beliefs regarding the severity of IA, the current study showed that more than half $(54.3 \%)$ of the participants disagreed with the severity of IA. This result contradicted Lau et al. (2018) who found that about half (48\%) of the participants agreed with the severity of IA. This contradiction might be attributed to the poor level of knowledge about IA among participants of the current study.

Concerning the participants' beliefs regarding the barriers to reduce internet time, the current study revealed that about near to half $(46 \%)$ of the participants agreed that feeling bored without the internet is a barrier for reducing internet time. This finding was congruent with Lau et al. (2018) who detected that about half (47\%) of the participants had the same belief. Another important barrier for reducing internet time perceived by the participant of the current study is that the internet is the main way for relieving stress in their life, while that barrier is agreed on by about half (49\%) of the participants. Based on a study explored the multidimensional needs of students for the prevention of IA by Shahrbabaki et al. (2017) adequate societal support such as designing entertainment programs for students or organizing sports events can help overcome these barriers.

Regarding the participants' perceived benefits of decreasing the internet time, the current study revealed that about two thirds (61.7\%) of the participants agreed that an important benefit of reducing internet time is the positive effect on their academic study. Similarly, Lau et al. (2018) revealed that about half $(47 \%)$ of the participants agreed that improving academic performance is a benefit of reducing internet use. Concerning the perceived self-efficacy toward 
reducing internet time, the current study revealed that more than two-thirds $(67.3 \%)$ of the participants disagreed with the easiness of reducing internet time. Contrary to the current study, Wang et al. (2016) revealed that more than half $(55.8 \%)$ of the participants disagreed with the difficulty of reducing the internet use.

According to Young (1998) IAT, the current study showed that $13 \%$ of the participants were normal internet users, less than half $(44.6 \%)$ had a mild level of IA followed by more than one third $(39.9 \%)$ had a moderate IA, while the minority $(3.5 \%)$ had a severe level of IA. These findings were in harmony with Khalil et al. (2016) who detected that more than one third $(38.4 \%)$, and $2.1 \%$ of participants were categorized as moderate to severe internet addict respectively while almost two-thirds $(59.6 \%)$ of the participant students were average (normal and mild) internet user. Similar results to the current study were found in a study conducted by Rajeswari et al. (2017) who found that less than one quarter $(22 \%)$ were normal internet user, about half $(49 \%)$ of the participants were mildly addicted to the internet followed by less than one third (28.5\%) moderately addicted, and the minority $(0.5 \%)$ severely addicted to the internet.

Regarding the relation between the level of IA and sociodemographic data of the participants, the present study showed that there was a significant statistical difference between the level of IA and gender of the participants, while males had a significant sever level of IA compared to females. This result were in the same line with Chi et al. (2020); Rajeswari et al. (2017); Krishnamurthy and Chetlapalli (2015); Anand et al. (2018) \& Ragheb et al. (2018).

The current study revealed that there was a significant statistical difference between the level of IA and the faculty grade of the participants. This result was in harmony with Abdelghani et al. (2018) who showed that there were significant differences between the average internet user and at-risk internet users in terms of academic grade. Another result agreed with the current result was revealed by Rajeswari et al. (2017). Contrary to the present study, Ragheb et al. (2018) in Egypt revealed there was no statistically significant association between IA and academic year of the participants.

The present study raveled that there were significant statistical differences between the level of IA and monthly family income of the participants while participants with a monthly family income ranged from 2000-3000 LE have a significant severe level of IA. The better socioeconomic status of the family may play a key role as sons are more likely to enjoy all the luxury that the world has to offer. These results agreed with Agnihotri et al. (2019); Abdelghani et al. (2018) $\&$ Xin et al. (2018).

The current study revealed that there was a significant statistical difference between the level of IA and academic performance of the participants while participants whose academic performance was pass/weak had a significant severe level of IA. These results agreed with Al-Hantoushi and Al-Abdullateef (2014)\& Stavropoulos et al. (2013) \& Iyitoglu and Celikoz (2017) \& Ambad et al. (2017). It could be theorized that participants with high IA levels stay more time online at the expense of their study duties given the enjoyment resulting from indulging in their favorite activities. Contrary to the current result, Usman et al. (2014) \&Ragheb et al. (2018) \& Kakaraki et al. (2017) \& McCamey et al. (2015) \& Najmi et al. (2014) indicated that there was no significant relationship between IA and academic achievement among participants.

\section{Conclusion}

The majority of participants had poor IA knowledge and low HBM constructs (perceived susceptibility, perceived severity, perceived benefits, and perceived self-efficacy) toward IA except for perceived barriers. The majority had mild and moderate levels of IA and the minority had a severe IA. The study also revealed a relationship between the level of IA and gender, faculty grade, monthly family income, and academic performance of the participants.

\section{Recommendations}

(1) 1-Strategies and different treatment modalities should be developed to increase awareness and decrease the level of IA among university students. For instance, Cognitive-behavioral therapy (CBT) and motivational interviewing are suggested by several studies as an effective treatment for IA.

(2) 2-Establishing more recreational services by the university such as sports centers to participate in hobbies can be helpful to defeat feelings of isolation, boredom, and symptoms of IA withdrawal.

(3) Nurse teachers need to include the different types of addictions, such as IA, to nursing study courses, and updated education on the issue is required.

(4) 4-Further studies include the participants' families in the intervention of IA is recommended especially, for students with severe level of IA to emphasize novel methods of socialization and pleasure for the whole family to increase their activities while offline.

\section{References}

(1) Abdelghani, M., El-Deen, G. S., Said, M., El-Gohary, H. M., Youssef, U. M., \& Bassiony, M. M. (2018). Determinants of Internet Addiction in a Sample of Egyptian University Students: A Survey. INTERNATIONAL ADDICTION REVIEW, 30.

(2) Agnihotri,L., Patil,N., Havinal,G., Balaganur,s., Mulla, A., Langoti, S. (2019)"a study to assess the IA level and effectiveness of PTP on adverse effect of IA and its management among engineering college students in selected college at vijayapur"Indian Journal of Applied research 9 (2).

(3) Al-Hantoushi, M., \& Al-Abdullateef, S. (2014). IA among secondary school students in Riyadh city, its prevalence, correlates and relation to depression: A questionnaire survey. Int J Med Sci Public Health, 3(1), 10-5.

(4) Ambad, S., Kalimin, K., \& Yusof, K. (2017). The effect of IA on students' emotional and scholastic performance. e-Academia Journal, 6(1), 86-98.

(5) Anand, N., Jain, P. A., Prabhu, S., Thomas, C., Bhat, A., Prathyusha, P. V., .. \& Cherian, A. V. (2018). Internet use patterns, internet addiction, and psychological distress among engineering university students: A study from India. Indian journal of psychological medicine, 40(5), 458-467.

(6) Chander,P. A Study To Assess The Effectiveness of A Structured Teaching Program Regarding Negative Impact of IA Among Adolescent In Selected Nursing College At Chandigarh. IOSR Journal of Nursing and Health Science (IOSR-JNHS) e-ISSN: 2320-1959.p- ISSN: 2320-1940 Volume 8, Issue 3 Ser. II. (May. $\begin{array}{llll}\text { June } & \text {.2019), } & \text { PP }\end{array}$ www.iosrjournals.or

(7) Chi, X., Hong, X., \& Chen, X. (2020). Profiles and sociodemographic correlates of Internet addiction in early adolescents in southern China. Addictive Behaviors, 106385.

(8) Cho, H., Kwon, M., Choi, J. H., Lee, S. K., Choi, J. S., Choi, S. W., \& Kim, D. J. (2014). Development of the Internet addiction scale based on the Internet Gaming Disorder criteria suggested in DSM-5. Addictive behaviors, 39(9), 1361-1366. 
(9) Dongyun, M., Ni'na, L., \& Yao, G. (2018). Relationship between impulsive personality traits and cognitive function in college students with internet addiction disorder. China Journal of Health Psychology, 1, 029.

(10) Eltigani (2019): Almost $80 \%$ of Internet Users in Egypt Access the Internet via Mobile Available at https://infotimes.org/almost80-of-internet-users-in-egypt-access-the-internet-via-mobileinternet/

(11) Fradelos, e. C., kourakos, m., velentza, o., polykandriotis, t., \& papathanasiou, i. V. (2016).internet addiction in children and adolescents: etiology, signs of recognition and implications in mental health nursing practice. Medico research chronicles 3: 264272.

(12) Griffiths, M. D. (2018). "Conceptual issues concerning internet Addiction and internet gaming disorder: Further critique on Ryding and Kaye (2017)." International Journal of mental health and Addiction 16(1): 233-239

(13) Hahn, E., Reuter, M., Spinath, F. M., \& Montag, C. (2017). Internet addiction and its facets: The role of genetics and the relation to self-directedness. Addictive Behaviors, 65, 137-146.

(14) Hamzaa, H. G. (2017). "The Internet Use and Addiction by University Adolescent Student." Port Said Scientific Journal of Nursing 4(1): 62-75.

(15) Hawi N (2013). Arabic validation of the Internet Addiction Test, Cyberpsychology, Behavior, and Social Networking; 16(3): 200204.

(16) Iyitoglu, O., \& Çeliköz, N. (2017). Exploring the impact of IA on academic achievement. European Journal of Education Studies, $3(5), 38-59$.

(17) Kakaraki, S., Tselios, N., \& Katsanos, C. (2017). IA, scholastic performance and personality traits: A correlational study among female university students. International Journal of Learning Technology, 12(2), 151-164

(18) Khalil, A. I., Alharbi, N. B., Alhawasawi, H. Y., \& Albander, A. B. (2016). Prevalence of IA among nursing students and the association with their academic performance and mental health. Athens Journal of Health, 3(4), 291-306.

(19) Krishnamurthy S, Chetlapalli SK.(2015). IA Prevalence and risk factors: A cross-sectional study among college students in Bengaluru, the Silicon Valley of India. Indian J Public Health 2015;59:115-21

(20) Kuss, D., D Griffiths, M., Karila, L., \& Billieux, J. (2014). Internet addiction: A systematic review of epidemiological research for the last decade. Current pharmaceutical design, 20(25), 4026-4052

(21) Lau, J. T., Wu, A. M., Cheng, K. M., Tse, V. W., Lau, M. M., \& Yang, X. (2018). Prevalence and factors of self $\square$ corrective intention among Hong Kong secondary school students who are self $\square$ assessed Internet addiction cases. Child and Adolescent Mental Health, 23(3), 155-163.

(22) Maheri, A., Tol, A., \& Sadeghi, R. (2017). Assessing the effect of an educational intervention program based on Health Belief Model on preventive behaviors of internet addiction. Journal of education and health promotion, 6

(23) Maheri, M., Nematollahi, S., Darabi, F., Bahrami, M. N., Moshki, M., \& Joveini, H. (2018). Knowledge, attitude, and self-efficacy regarding internet addiction among female students. Journal of Research and Health, 8(5), 459-465.

(24) McCamey, R., Wilson, B., \& Shaw, J. (2015). Internet dependency and scholastic performance. The Journal of Social Media in Society, 4, 126-150
(25) MMIN, M. (2017). "A descriptive study to assess the knowledge and attitude regarding internet usage and its addiction level among students studying in selected colleges of ambala, haryana."

(26) Najmi, H. U., Masoumeh, A., \& Syed, M. (2014). Relationship between IA and scholastic performance among foreign undergraduate students. Social and Behavioral Sciences, 114, 845851.

(27) Ong, S. H. and Y. R. Tan (2014). "Internet addiction in young people." Ann Acad Med Singapore 43(7): 378-382.

(28) Orji, R., Vassileva, J., \& Mandryk, R. (2012). Towards an effective health interventions design: an extension of the health belief model. Online journal of public health informatics, 4(3).

(29) Petry, N. M. and C. P. O'Brien (2013). "Internet gaming disorder and the DSM- 5." Addiction 108(7): 1186-1187.

(30) Ragheb, B. M., El-Boraie, O. A., Shohda, M. M., \& Ibrahim, N. (2018) Internet Addiction and Quality of Life among Students at Technical Institute of Nursing, Mansoura University, Egypt.

(31) Rajeswari, C., Joseph, N., George, N., Syhly, P., \& Jose, P. (2017) Internet addiction among the undergraduate students. Nitte University Journal of Health Science, 7(1).

(32) Shahrbabaki, B. N., Fallahi, A., \& Pirakalathanan, P. (2017). Expressed Needs of Students for Prevention of Internet Addiction: A Content Analysis Study. Health Scope, 6(3).

(33) Smyth, S. J., Curran, K., \& McKelvey, N. (2019). Internet Addiction: The Repercussions, the Causes, and the Treatment. In Multifaceted Approach to Digital Addiction and Its Treatment (pp. 1-19). IGI Global.

(34) Spada, M. M. (2014). "An overview of problematic Internet use." Addictive behaviors 39(1): 3-6.

(35) Stavropoulos, V., Alexandraki, K., \& Motti-Stefanidi, F. (2013). Recognizing Internet addiction: Prevalence and relationship to academic achievement in adolescents enrolled in urban and rural Greek high schools. Journal of Adolescence, $36,565-576$.

(36) Thakur, A., Peepre, K., Vaswani, A., Gupta, K., Verma, A., Singh, D., \& Kasar, P. (2018). Internet addiction, behavioral aspects and health-related problems associated with it: A cross-sectional study among engineering students of Jabalpur district. Int J Res Med Sci, 6(1), 253-8.

(37) Usman, N., Alavi, M., \& Shafeq, S. M. (2014). Relationship between internet addiction and academic performance among foreign undergraduate students. Procedia-Social and Behavioral Sciences, $114,845-851$.

(38) Wang, Y., Wu, A. M., \& Lau, J. T. (2016). The health belief model and number of peers with internet addiction as inter-related factors of Internet addiction among secondary school students in Hong Kong. BMC Public Health, 16(1), 272.

(39) Wiederhold, B. K. (2018). Stop scrolling, start living: the growing reality of internet addiction disorder.

(40) Xin, M., Xing, J., Pengfei, W., Houru, L., Mengcheng, W., \& Hong, Z. (2018). Online activities, prevalence of Internet addiction and risk factors related to family and school among adolescents in China. Addictive Behaviors Reports, 7, 14-18.

(41) Young, K. S. (1998). Internet addiction: The emergence of a new clinical disorder. Cyberpsychology \& behavior, 1(3), 237-244.

(42) Zadeh, D. S., Changizi, M., \& Sadegh, R. (2014). The effect of education about addiction through health belief model (HBM) on knowledge and perceptions of high school students in Shadegan. J Sci Todays World 2014;3:240-4 